\title{
ON INFINITELY DIVISIBLE LAWS IN $C[0,1]$
}

\author{
ALOISIO PESSOA DE ARAUJO ${ }^{1}$
}

ABSTRACT. In Euclidean spaces, or in a separable Hilbert space, the characteristic function of an infinitely divisible distribution has the familiar form given by the Lévy-Khintchine formula. The Lévy measures $M$ of this formula are characterized by the property that the integral of $\min \left[1,\|x\|^{2}\right]$ with respect to $M$ is finite. This simple situation no longer holds in the Banach space $C=C[0,1]$ where integrability of $\min [1,\|x\|]$ is sufficient but integrability of $\min \left[1,\|x\|^{2}\right]$ is neither necessary nor sufficient.

Certain other conditions which are sufficient to imply that $M$ is the Lévy measure of a distribution on $C$ can be obtained with the use of an integral formula of Garsia.

1. Introduction. Let $C$ be the Banach space $C=C[0,1]$ of continuous functions on the interval $[0,1]$ with the uniform norm. Let $M$ be a positive measure on $C$. Suppose that for each element $\mu$ of the dual $C^{\prime}$ of $C$ the integral $\int \min \left\{1,|\langle\mu, x\rangle|^{2}\right\} M(d x)$ is finite. Let $h$ be the function

$$
\left.h(\mu, x)=\exp \{i\langle\mu, x\rangle\}-1-i\langle\mu, x\rangle\left[1+|\langle\mu, x\rangle|^{2}\right]^{-1}\right\} \text {. }
$$

Define $\psi$ on $C^{\prime}$ by

$$
\psi(\mu)=\exp \int h(\mu, x) M(d x)
$$

It can be shown that such a $\psi$ is always the Fourier transform of an infinitely divisible distribution $P$ carried by the algebraic dual of $C^{\prime}$. We shall call $M$ a proper Lévy measure if the probability measure $P$ is carried by $C$ itself. The literature does not seem to contain any proof of the fact that every infinitely divisible distribution $P$ carried by $C$ is the convolution of a Gaussian measure by a measure whose Fourier transform has the form (2). This is true, as we shall show elsewhere, and is well known for certain special Banach spaces other than $C$. For instance, if $C$ were replaced by a separable Hilbert space, one would know from Parthasarathy [1] that infinitely divisible distributions have a Lévy-Khintchine representation. In addition, we would know from Varadhan [2] that a necessary and sufficient

Received by the editors June 10, 1974.

AMS (MOS) subject classifications (1970). Primary 60F05; Secondary 60G99.

1 This research was supported partially by U. S. Army Research Office (Durham) Grant DA-ARO-D-31-124-G1135 and Conselho Nacional de Pesquisas, Brasil. 
condition for a positive measure $M$ to be a proper Lévy measure is that $\int \min \left[1,\|x\|^{2}\right] M(d x)<\infty$.

We shall proceed to show, in $\$ 2$ below, that $\int \min [1,\|x\|] M(d x)<\infty$ is always a sufficient condition, but give counterexamples showing that in $C$ the integrability of $\min \left[1,\|x\|^{2}\right]$ is neither necessary nor sufficient. Extensions to functions of the norm which decrease faster than $\|x\|$ are possible and will be given in another publication. This shows that integrability conditions on functions of the norm are not necessarily the appropriate criteria. We proceed in $\$ 3$ to give certain conditions which are sufficient to imply that $M$ is a proper Lévy measure, using a formula of Garsia, Rodemich and Rumsey [3]. The results of this nature can also be rephrased in terms of entropy conditions such as used in the Gaussian case by Dudley [4]. They lead naturally to sufficient conditions for central limit theorems in $C[0,1]$.

2. Integrability of functions of the norm. Let $M$ be a positive measure in a separable Banach space. Necessary and sufficient conditions for $M$ to be a proper Lévy measure, that is, the Lévy measure of a distribution carried by the Banach space, are known in the finite dimensional or Hilbertian case where they take the form $\int \min \left[1,\|x\|^{2}\right] M(d x)<\infty$. We first show that such a condition is neither necessary nor sufficient in $C=C[0,1]$. The examples will use two sequences $\left\{X_{n}\right\}$ and $\left\{X_{n}^{\prime}\right\}$ of independent Poisson random variables such that $E X_{n}=\lambda_{n}=E X_{n}^{\prime}$, defined on a probability space $\Omega$, and sequences $\left\{g_{n}\right\}, g_{n} \in C,\left\|g_{n}\right\| \leq 1$. When the series converges in probability for each $\mu \in C^{\prime}$, the sum

$$
\langle\mu, Y\rangle=\sum_{n}\left(X_{n}-X_{n}^{\prime}\right)\left\langle\mu, g_{n}\right\rangle
$$

defines a stochastic process. Its characteristic function has the form

$$
E \exp \{i\langle\mu, Y\rangle\}=\exp \int[\exp \{i\langle\mu, x\rangle\}-1] M(d x)
$$

for a Lévy measure $M$ which assigns masses $\lambda_{n}$ to each of $\left\{g_{n}\right\}$ and $\left\{-g_{n}\right\}$ for each $n$.

Proposition 1. In the space $C$, there are sequences $\left\{\lambda_{n^{\prime}} g_{n}\right\}$ such that $M$, defined above, is a proper Lévy measure, but $\int \min \left[1,\|x\|^{2}\right] M(d x)=\infty$.

Proof. Let $\lambda_{n}=n^{-3 / 4}$ and $c_{n}=n^{-1 / 8}$. Let $f_{n}$ be the function defined by $f_{n}\left[3 \cdot 2^{-(n+1)}\right]^{n}=1, f_{n}(s)=0$ if $s \notin I_{n}=\left(2^{-n}, 2^{-(n-1)}\right)$, and linear interpolation in between. Take for $g_{n}$ in formula (3) the function $g_{n}=c_{n} f_{n}$. Then $\int\|x\|^{2} M(d x)=2 \Sigma c_{n}^{2} \lambda_{n}=\infty$. 
To show that $M$ is nevertheless a proper Lévy measure consider the sum $Y(s, \omega)=\Sigma_{n}\left[X_{n}(\omega)-X_{n}^{\prime}(\omega)\right] g_{n}(s)$. For $s>0$ this is continuous since it is a finite sum. Also

$$
\sum P\left\{\left|X_{n}-X_{n}^{\prime}\right|>1\right\} \leq 2 \sum P\left[X_{n}>1\right] \leq 4 \sum \lambda_{n}^{2}<\infty .
$$

Thus, according to the Borel-Cantelli theorem, for almost every $\omega \in \Omega$ there is an integer $n(\omega)$ such that $n \geq n(\omega)$ implies $\left|X_{n}(\omega)-X_{n}^{\prime}(\omega)\right| \leq 1$. For $n$ $\geq n(\omega)$ and $s \in I_{n}$ this gives the inequality $|Y(s, \omega)| \leq\left|X_{n}(\omega)-X_{n}^{\prime}(\omega)\right| g_{n}(s)$ $\leq n^{-1 / 8}$. Since $Y(s, \omega)=0$, this implies the almost sure continuity of $Y$ and the convergence of the series which defines $\langle\mu, Y\rangle$, concluding the proof of the proposition.

Proposition 2. There are sequences $\left\{\lambda_{n}, g_{n}\right\}$ such that $Y(s, \omega)=$ $\Sigma\left[X_{n}(\omega)-X_{n}^{\prime}(\omega)\right] g_{n}(s)$ is almost surely an unbounded function of $s$, even though $\int\|x\|^{2} M(d x)<\infty$.

Proof. Let $\lambda_{n}=1, n=1,2, \ldots$. For $j=1,2, \ldots$, let $a_{n}$ be equal to $j^{-1} 2^{-j / 2}$ if $2^{j} \leq n<2^{j+1}$. Take for function $g_{n}$ the expression $g_{n}(s)=$ $a_{n} \cos \left(2 \pi n s+b_{n}\right)$. Then $\int\|x\|^{2} M(d x)=\Sigma_{j} j^{-2}<\infty$. Also, the series defining $\langle\mu, Y\rangle$ converges in quadratic mean since $\Sigma a_{n}^{2}<\infty$. We claim that the constant $b_{n}$ may be chosen so that for almost all $\omega$ one has $\sup _{s}|Y(s, \omega)|=\infty$. To prove this note that if $s_{j}^{2}=\Sigma_{n}\left\{a_{n}^{2} ; 2^{j} \leq n<2^{j+1}\right\}$ then $\Sigma_{j} s_{j}=\infty$ and consider the sums

$$
Z(s, \omega)=\sum_{n} a_{n}\left[X_{n}(\omega)-X_{n}^{\prime}(\omega)\right] \cos \left[2 \pi n s+\phi_{n}(\omega)\right]
$$

where the $\phi_{n}(\omega)$ are uniformly distributed on $[0,2 \pi)$ independently of each other and of the sequences $\left\{X_{n}\right\},\left\{X_{n}^{\prime}\right\}$. A lemma of Paley and Zygmund (see for instance Kahane [5, p. 85]) says that the function $s \rightarrow Z(s, \omega)$ is almost surely not an element of $L^{\infty}$. By Fubini's theorem this remains true for almost all values of the $\phi_{n}(\omega)$ implying the existence of sequences $\left\{b_{n}\right\}$ for which $s \rightarrow Y(s, \omega)$ is almost surely not an element of $L^{\infty}$.

In such a case $M$ is a Lévy measure for a process on the algebraic dual of $C^{\prime}$, but not for a distribution on $C$ or even on the second dual $C^{\prime \prime}$.

LeCam suggested (private communication) that the construction of Proposition 2 can be modified to yield the following. Suppose that $r$ is a nonnegative function defined on $[0, \infty)$ and such that $\lim u^{-1} r(u)=0$ as $u \rightarrow 0$. Then there are positive measures $M$ on $C$ which are not proper Lévy measures even though they satisfy the requirement that $\int r[\|x\|] M(d x)<\infty$. 
In view of this, the following result gives the best sufficient condition in terms of integrability of functions of the norm.

Theorem 1. Let $M$ be a positive Borel measure on a separable Banach space. If $\int \min [1,\|x\|] M(d x)<\infty$ then $M$ is the Lévy measure of an infinitely divisible distribution carried by the Banach space.

Proof. Let $M_{n}$ be the restriction of $M$ to the set $R_{n}=\{x ;(1 /(n+1))<$ $\|x\| \leq(1 / n)\}$. Then $\left\|M_{n}\right\|$ is finite.

Let $Z_{n}=\Sigma_{j \leq N_{n}} Y_{n, j}$ where all the variables $N_{n}, Y_{n, j}, j=1,2, \ldots$, are independent, $N_{n}$ has a Poisson distribution with $E N_{n}=\left\|M_{n}\right\|$ and the $Y_{n, j}$ have the distribution $M_{n} /\left\|M_{n}\right\|$. Then $\left\|Z_{n}\right\| \leq \Sigma_{j_{-} N_{n}}\left\|Y_{n, j}\right\|$ and therefore

$$
E\left\|Z_{n}\right\| \leq\left(E N_{n}\right) E\left\|Y_{n, j}\right\| \leq\left\|M_{n}\right\| n^{-1} \text {. }
$$

If $\int \min [1,\|x\|] M(d x)<\infty$, the series $\Sigma Z_{n}$ converges in $L_{1}$-norm. Hence the restriction of $M$ to the unit ball is a proper Lévy measure. The part of $M$ situated outside the unit ball is finite. It is also a Lévy measure. Hence the result.

3. A sufficient condition and some of its applications. Conditions which imply that $M$ is a proper Lévy measure can be obtained through an inequality of Garsia, Rodemich and Rumsey [3].

Before stating them let us note the easily verified fact that $M$ is a proper Lévy measure if and only if the symmetrized measure $M_{s}$ defined by $M_{s}(A)$ $=M(A)+M[(-A)]$ is likewise a proper Lévy measure. In view of this we shall concentrate on symmetric measures.

The following notation will be used. Let $\rho$ be a continuous increasing function defined on $[0, \infty)$. Assume that $\rho(0)=0$ and let $\rho(u)=\rho(|u|)$ for $u<0$. For every $f \in C[0,1]$ let

$$
\phi(s, t ; f)=[f(s)-f(t)][\rho(s-t)]^{-1}
$$

and let $B(f)$ be the integral

$$
B(f)=\int_{0}^{1} \int_{0}^{1} \exp \{|\phi(s, t ; f)|\} d s d t .
$$

Finally, if $M$ is a positive measure on $C[0,1]$ let $J(s, t ; M)$ be the integral

$$
J(s, t ; M)=\int\{\exp [\phi(s, t ; f)]-1-\phi(s, t ; f)\} M(d f) .
$$

Theorem 2. Assume that the function $\rho$ is such that 
(i) $\int(\rho(u) / u) d u<\infty$.

For a fixed $b<\infty$ let $M$ be the set of all $\sigma$-finite positive symmetric measures such that

(ii) $\int f^{2}(0) M(d f) \leq b$,

(iii) $\int_{0}^{1} \int_{0}^{1} \exp \{J(s, t ; M)\} d s d t \leq b$,

then each $M \in \mathbb{M}$ is the Lévy measure of an infinitely divisible distribution $P_{M}$ on $C=C[0,1]$ and the set $\left\{P_{M} ; M \in \mathbb{M}\right\}$ is tight on $C$.

Proof. Consider first a finite $M \in M$. This is the Lévy measure of a distribution $P_{M}$ for which

$$
\int \exp \{|\phi(s, t ; f)|\} P_{M}(d f) \leq 2 \int \exp \{\phi(s, t ; f)\} P_{M}(d f) \leq 2 \exp \{J(s, t ; M)\} .
$$

Take a $\beta>0$ and let $S_{\beta}$ be the bounded equicontinuous set of all $f \in C$ such that $|f(0)|^{2} \leq \beta$ and

$$
|f(t)-f(s)| \leq 8 \int_{0}^{|t-s|} \log \left(\beta / u^{2}\right) d \rho(u),
$$

for all $(s, t)$. According to [3] the inequalities $B(f) \leq \beta$ and $|f(0)|^{2} \leq \beta$ imply that $f \in S_{\beta}$. The assumptions (ii) and (iii) and Markov's inequality yield immediately that $P_{M}\left(S_{\beta}\right) \geq 1-3(b / \beta)$. Hence the result for the finite $M \in \mathbb{M}$. An arbitrary $M \in \mathbb{M}$ is the limit of an increasing sequence $M_{n}$ of finite elements of $M$. The tightness obtained in the first part of the argument shows then that $P_{M_{n}}$ converges to a $P_{M}$ whose Lévy measure is $M$. This completes the proof of the theorem.

Corollary. Let $M$ be a positive, symmetric measure on a compact subset $K$ of $C[0,1]$. Suppose that there is an even continuous function $q$ defined on $[-1,+1]$ and increasing on $[0,1]$ such that $|f(s)-f(t)| \leq q^{2}(s-t)$ for all pairs $(s, t)$ and all $f \in K$.

Assume that for some $a \in(0,1)$ one has

(a) $\int\|f\|^{2-a} M(d f)<\infty$,

(b) $\int_{0}^{1}[q(u)]^{a}|\log (u / 2)|^{-1 / 2}(d u / u)<\infty$.

Then $M$ is a proper Lévy measure.

Proof. Let $c$ be a number such that $c^{2}>2 e \int\|f\|^{2-a} M(d f)$. Define a function $\rho$ by $\rho(u)=c q^{a}(u)|\log (u / 2)|^{-1 / 2}$. It can be verified that condition (b) implies that such a $\rho$ satisfies the requirements imposed for Theorem 2 . Define $\phi(s, t ; f)$ as before and consider first the set $A$ of all $f \in K$ such that 
$|\phi(s, t ; f)| \leq 1$ for all pairs $(s, t)$. Let $M_{1}$ be the measure $M$ restricted to this set.

The integral $J\left(s, t ; M_{1}\right)$ can be bounded by

$$
J\left(s, t ; M_{1}\right) \leq \frac{1}{2} e \int|\phi(s, t ; f)|^{2} M(d f)
$$

Also

$$
\begin{aligned}
|\phi(s, t ; f)|^{2} & \leq 4\|f\|^{2-a}|f(s)-f(t)|^{a} \rho^{-2}(s-t) \\
& \leq 4 c^{-2}\|f\|^{2-a}|f(s)-f(t)|^{a} q^{-2 a}(s-t)|\log 1 / 2| s-t|| \cdot .
\end{aligned}
$$

Therefore

$$
J\left(s, t ; M_{1}\right) \leq 4 e c^{-2}\left[\int\|f\|^{2-a} M(d f)\right]|\log 1 / 2| s-t \| .
$$

Since in this expression the coefficient of the logarithmic term is strictly less than unity we conclude that $\int_{0}^{1} \int_{0}^{1} \exp \left\{J\left(s, t ; M_{1}\right)\right\} d s d t<\infty$. Hence $M_{1}$ is a proper Lévy measure by Theorem 2 .

To complete the proof it is sufficient to show that $M\left(A^{c}\right)<\infty$, since $M$ restricted to $A^{c}$ will then be a proper Lévy measure.

For this purpose take a $k>2$ and write $\Delta=|f(t)-f(s)|$ and $q=q(s-t)$ for short. Then

$$
\begin{aligned}
|c| f(t)-f(s)\left|\rho^{-1}(s-t)\right|^{k} & =\Delta^{2-a} \Delta^{k-(2-a)} q^{-k a}[\log 1 / 2|s-t|]^{k / 2} \\
& \leq(2\|f\|)^{2-a} q^{2[k-(2-a)]-k a}|\log 1 / 2| s-\left.t\right|^{k / 2} .
\end{aligned}
$$

The condition (b) implies that $q^{a}(u)|\log (u / 2)|^{1 / 2}$ tends to zero as $u \rightarrow 0$. Therefore if $k(1-\alpha)>2-\alpha$, we can assert that

$$
|c| f(t)-f(s)\left|\rho^{-1}(s-t)\right|^{k} \leq c_{1}\|f\|^{2-a}
$$

for all $(s, t)$ and a suitable constant $c_{1}$. The finiteness of $M\left(A^{c}\right)$ follows by Markov's inequality.

Examples. (1) Let $\rho_{2}(u)$ be the function defined by letting $r^{2}(s, t)=$ $\int(f(s)-f(t))^{2} M(d f)$ and $\rho_{2}(u)=\sup \{r(s, t) ;|s-t| \leq u\}$. Suppose that $M=$ $\sum_{n=1}^{\infty} \lambda_{n}\left(\delta_{\left\{f_{n}\right\}}+\delta_{\left\{-f_{n}\right\}}\right)$ with $\lambda_{n}>c>0, f_{n} \in C[0,1]$. Then condition (iii) of Theorem 2 is satisfied with $\rho(u)=\rho_{2}(u)$.

(2) Using the same method as in the Corollary one can show that $M$ is a proper Lévy measure if there is a $\rho$ satisfying the conditions of Theorem 2 such that one has always $|f(s)-f(t)| \leq c_{f}(s, t) \rho(s-t)$ with 


$$
\int \sup _{f}\left\{c_{f}^{2}(s, t) ;|s-t|<u\right\} M(d f)<\infty
$$

for some $u \in(0,1)$.

Remarks. (1) Theorem 2 can easily be extended to the space of continuous functions on a $k$-dimensional cube, or other compact metric spaces, using the results of Garsia [7] and Preston [8].

(2) When checking condition (iii) of Theorem 2 one can always remove a part $M^{\prime}$ of $M$ such that $\int \min [1,\|x\|] M^{\prime}(d x)<\infty$, since the sum of two proper Lévy measures is a proper Lévy measure.

(3) Our application of Garsia's formula relies on the function exp $u$ instead of the $\exp u^{2}$ used for Gaussian variables, since introducing the square may easily make the expectations infinite.

(4) It is known (see LeCam [6]) that tightness of sets of infinitely divisible distributions may be used to prove central limit theorems. This will be investigated in another publication.

\section{REFERENCES}

1. K. R. Parthasarathy, Probability measures on metric spaces, Probability and Mathematical Statistics, No. 3, Academic Press, New York, 1967. MR 37 \#2271.

2. S. R. S. Varadhan, Limit theorems for sums of independent random variables with values in a Hilbert space, Sankhyā Ser. A 24 (1962), 213-238. MR 30 \#1536.

3. A. M. Garsia, E. Rodemich and $\mathrm{H}$. Rumsey, Jr., A real variable lemma and the continuity of paths of some Gaussian processes, Indiana Univ. Math. J. 20 (1970/71), 565-578. MR $42 \# 2534$.

4. R. M. Dudley, The sizes of compact subsets of Hilbert space and continuity of Gaussian processes, J. Functional Analys is 1 (1967), 290-330. MR 36 \#3405.

5. J.-P. Kahane, Séries de Fourier aléatoires, 2nd ed., Séminaire de Math. Supérieures, No. 4 (1963), Univ. de Montréal, Montréal, 1967. MR 42 \#3483.

6. L. M. LeCam, Remarques sur le théorème limite central dans les espaces localement convexes, Les Probabilités sur les Structures Algébriques, C.N.R.S., Paris, 1970.

7. A. M. Garsia, Continuity properties of Gaussian process with multidimensional time parameter, Proc. Sixth Berkeley Sympos. on Math. Stat. and Prob., Vol. 2, University of California Press, Berkeley, 1972, pp. 369-374.

8. C. Preston, Continuity properties of some Gaussian processes, Ann. Math. Statist. 43 (1972), 285-292. MR 46 \#6436.

DEPARTMENT OF STATISTICS, UNIVERSITY OF CALIFORNIA, BERKELEY, CALIFORNIA 94720 